\title{
Cofinite hyperbolic Coxeter groups, minimal growth rate and Pisot numbers
}

\author{
RUTH KELLERHALS
}

\begin{abstract}
By a result of $\mathrm{R}$ Meyerhoff, it is known that among all cusped hyperbolic 3-orbifolds the quotient of $\mathbb{H}^{3}$ by the tetrahedral Coxeter group $(3,3,6)$ has minimal volume. We prove that the group $(3,3,6)$ has smallest growth rate among all non-cocompact cofinite hyperbolic Coxeter groups, and that it is as such unique. This result extends to three dimensions some work of W Floyd who showed that the Coxeter triangle group $(3, \infty)$ has minimal growth rate among all non-cocompact cofinite planar hyperbolic Coxeter groups. In contrast to Floyd's result, the growth rate of the tetrahedral group $(3,3,6)$ is not a Pisot number.
\end{abstract}

20F55; 22E40, 51F15

\section{Introduction}

Let $\mathbb{H}^{n}$ denote the standard hyperbolic $n$-space. A Coxeter polytope $P \subset \mathbb{H}^{n}$ is a convex polytope all of whose dihedral angles are of the form $\pi / k$ for an integer $k \geq 2$. We always assume that $P$ is of finite volume so that it is bounded by finitely many hyperplanes $H_{i}, i \in I$. The reflections $s_{i}$ with respect to $H_{i}, i \in I$, generate a discrete group $G$ of hyperbolic isometries which is a Coxeter group $G=(G, S)$ with presentation $\langle S \mid R\rangle$ where

$$
S=\left\{s_{i} \mid i \in I\right\}, \quad R=\left\{s_{i}^{2}=1,\left(s_{i} s_{j}\right)^{k_{i j}}=1 \mid i, j \in I, i \neq j\right\} .
$$

In (1-1), the exponents $k_{i j}$ are integers $\geq 2$, symmetric with respect to $i, j$, and related to the dihedral angles formed by $H_{i}, H_{j}$ when intersecting in $\mathbb{H}^{n}$. We often represent $G$ (and $P$ ) by its Coxeter graph $\Sigma$ or its Coxeter symbol if the presentation (1-1) for $G$ is simple enough (see Section 2.2).

In the focus of this work are non-compact Coxeter polyhedra $P \subset \mathbb{H}^{3}$ of finite volume which form a vast, infinite set (see Section 2.2). The Coxeter tetrahedron with graph

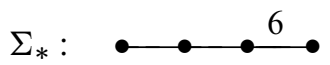

and Coxeter symbol $(3,3,6)$ is of particular importance. It is a building block for an ideal regular tetrahedron and has one vertex at infinity. It yields the 1-cusped 
quotient space $\mathbb{H}^{3} /(3,3,6)$ which has minimal volume among all cusped hyperbolic 3-orbifolds as proven by Meyerhoff [15].

We shall study another quantity related to hyperbolic Coxeter groups $G=(G, S)$, namely the growth rate $\tau_{G}$ associated to the growth series (see Section 3)

$$
f_{S}(x)=1+|S| x+\sum_{n \geq 2} a_{n} x^{n}, \quad x \in \mathbb{C},
$$

where $a_{n}$ denotes the number of words $w \in G$ of $S$-length equal to $n$. More precisely, the growth series $f_{S}$ of $G$ has radius of convergence $R<1$ and is the series expansion of a rational function $p(x) / q(x)$ with coprime elements $p(x), q(x) \in \mathbb{Z}[x]$. With this said, the growth rate $\tau_{G}$ is defined to be the reciprocal of $R$. It follows that $\tau_{G}>1$ is a root of maximal absolute value of $q(x)$ and an algebraic integer. As such $\tau_{G}$ is an interesting object in the realm of Salem numbers, Pisot numbers and Perron numbers (see Section 3.2). We shall prove the following main result of this work (see Section 4).

Theorem Among all hyperbolic Coxeter groups with non-compact fundamental polyhedron of finite volume in $\mathbb{H}^{3}$, the tetrahedral group $(3,3,6)$ has minimal growth rate, and as such the group is unique.

The Theorem completes the picture of growth rate minimality for cofinite hyperbolic Coxeter groups in three dimensions. Indeed, in [9], we showed that the growth rate of the group $(3,5,3)$ is minimal among all growth rates (being Salem numbers) of Coxeter groups acting cocompactly on $\mathbb{H}^{3}$.

Let us briefly discuss the proof of the Theorem. We exploit Steinberg's formula

$$
\frac{1}{f_{S}\left(x^{-1}\right)}=\sum_{\substack{G_{T}<G \\\left|G_{T}\right|<\infty}} \frac{(-1)^{|T|}}{f_{T}(x)}
$$

expressing $f_{S}\left(x^{-1}\right)$ in terms of the growth functions $f_{T}(x)$ of the finite subgroups $G_{T}$ of $G$. Each part $f_{T}(x)$ in (1-4) is, by Solomon's formula, a product of certain polynomials related to the Coxeter exponents of $T$ (see Section 3). Our first observation is that these exponents satisfy a certain monotonicity property (see Section 4.1). Although the function $f_{G}(x):=f_{S}(x)$ is - in presence of ideal vertices of $P$ - not anti-reciprocal anymore (see Section 3.1), we are able to prove

$$
\frac{1}{f_{G}(x)}<\frac{1}{f_{(3,3,6)}(x)} \quad \text { for all } \quad x \in\left(0,1 / \tau_{(3,3,6)}\right]
$$

for Coxeter groups $G$ different from $(3,3,6)$ in the following way. Since the ideal vertices of $P$ are either 3 -valent or 4 -valent (see Section 2.2), the set of vertices of $P$ 
can be partitioned according to $\Omega_{0}=\Omega_{f} \cup \Omega_{\infty}^{3} \cup \Omega_{\infty}^{4}$ where $\Omega_{f}$ is the set of finite (3-valent) vertices of $P$. If $\Omega_{\infty}^{4} \neq \varnothing$, a result of Kolpakov [11] shows that $\tau_{G}$ is the limit of an increasing sequence of growth rates $\tau_{G_{n}}$ for Coxeter groups $G_{n}$ having less 4-valent ideal vertices than $G$. This implies that we can restrict to the case $\Omega_{\infty}^{4}=\varnothing$ and consider simple Coxeter polyhedra, allowing us to identify $1 / f_{G}(x)$ in a new and very efficient way (see (4-9), (4-11) and (4-12)), and at the end, to verify (1-5).

Finally, the proof of the Theorem can be easily adapted to the two-dimensional case and provides an elementary verification of the result of Floyd [4] that the Coxeter triangle group $(3, \infty)$, which is closely related to the modular group $S L(2, \mathbb{Z})$, has minimal growth rate among all hyperbolic Coxeter groups with non-compact fundamental polygon of finite area in $\mathbb{H}^{2}$. Notice that our proof (see Section 5.2) does not rely upon the theory of Pisot polynomials, the identification of $\tau_{(3, \infty)}$ with the Pisot number $\alpha_{S} \simeq 1.324718$ of $x^{3}-x-1$, and the result of Smyth [18] that $\alpha_{S}$ is the smallest Pisot number (see Section 3.2). In this context, observe that the growth rate $\tau_{(3,3,6)}$ is not a Pisot number but a Perron number, at least (see Remark, Sections 5.1 and 5.2, and Komori and Umemoto [12]).

Acknowledgements The author was partially supported by Schweizerischer Nationalfonds 200020-131967 and 200020-144438. She would like to thank the Mittag-Leffler Institut in Djursholm, Sweden, for the warm hospitality during the preparation of this paper.

\section{Cofinite hyperbolic Coxeter groups}

\subsection{Convex hyperbolic polytopes}

Denote by $\mathbb{X}^{n}, n \geq 2$, either the standard hyperbolic $n$-space $\mathbb{H}^{n}$, the unit sphere $\mathbb{S}^{n}$, or the Euclidean space $\mathbb{E}^{n}$. Interpret $\mathbb{X}^{n} \neq \mathbb{E}^{n}$ in its vector space model, that is, $\mathbb{X}^{n}$ is a subset of a real vector space $\mathbb{Y}^{n+1}$ equipped with a bilinear form $\langle\cdot, \cdot\rangle$ inducing the metric structure on $\mathbb{X}^{n}$. In particular, we view hyperbolic space $\mathbb{H}^{n}$ as embedded in the Lorentz-Minkowski space $\mathbb{Y}^{n+1}=\mathbb{E}^{n, 1}$ of signature $(n, 1)$ so that points of the boundary $\partial \mathbb{H}^{n}$ are vectors of vanishing norm. A convex polytope $P \subset \mathbb{X}^{n}$ is defined to be the intersection of finitely many half-spaces bounded by hyperplanes $H_{i}, i \in I$, in $\mathbb{X}^{n}$, where each $H_{i}$ can be written as orthogonal complement of a vector $e_{i} \in \mathbb{Y}^{n+1}$ of positive norm, directed outwards with respect to $P$, say. In the sequel, we consider convex polytopes of finite volume, only. In the hyperbolic context, the finite volume condition is equivalent to the property that $P$ is the convex hull of finitely many points or vertices in $\mathbb{H}^{n} \cup \partial \mathbb{H}^{n}$. A vertex $v \in P$ lying in $\mathbb{H}^{n}$ is called a finite vertex, and a 
vertex $v_{\infty} \in \bar{P}$ lying on $\partial \mathbb{H}^{n}$ is called an ideal vertex of $P$. If all vertices of $P$ are finite, then $P$ is compact. If all vertices of $P$ are ideal, we call $P$ an ideal hyperbolic polytope. Consider the $\operatorname{Gram}$ matrix $\operatorname{Gram}(P)$ associated to the vectors $e_{i}, i \in I$, whose non-diagonal entries are metrically related to the dihedral angles and distances between the hyperplanes $H_{i}$ bounding $P$. In particular, a non-diagonal entry $g_{i j}$ of $\operatorname{Gram}(P)$, which is of absolute value smaller than one, can be interpreted according to

$$
g_{i j}=\left\langle e_{i}, e_{j}\right\rangle=-\cos \angle\left(H_{i}, H_{j}\right)
$$

that is, the hyperplanes $H_{i}, H_{j}$ intersect under the dihedral angle $\angle\left(H_{i}, H_{j}\right)$ in the face $F_{i j}=H_{i} \cap H_{j} \cap P$ of $P$. In [21, Chapter I] and [22, Part I, Chapter 6], Vinberg developed explicit criteria for the existence of an acute-angled polytope $P \subset \mathbb{H}^{n}$ in terms of the Gram matrix, as well as criteria for compactness, finite volume and vertices to be finite or ideal. As an example, a hyperbolic tetrahedron $S \subset \mathbb{H}^{3}$ with dihedral angles not bigger than $\pi / 2$ is characterised by a $4 \times 4$ matrix $G=\left(g_{i j}\right)$ with $g_{i i}=1$ such that the signature of $G$ equals $(3,1)$. A vertex $v \in S$ is finite if the principal submatrix $G_{v}$, formed by the three hyperplanes passing through $v$, is positive definite, while an ideal vertex $v_{\infty} \in \bar{S}$ is characterised by a principal submatrix $G_{v_{\infty}}$ which is positive semi-definite.

\subsection{Coxeter polytopes and Coxeter groups in $\mathbb{X}^{n}$}

A Coxeter polytope $P \subset \mathbb{X}^{n}$ is a convex polytope such that all its dihedral angles are submultiples of $\pi$, that is, they are of the form $\pi / k$ with an integer $k \geq 2$. We call two hyperplanes in $\mathbb{H}^{n}$ parallel resp. ultra-parallel if they meet on the boundary $\partial \mathbb{H}^{n}$ resp. if they admit a common perpendicular in $\mathbb{H}^{n}$ realising their distance. For a given Coxeter polytope $P \subset \mathbb{X}^{n}$, consider the group $G$ generated by the reflections $s_{i}$ in the hyperplanes $H_{i}$ bounding $P . G$ is called a geometric Coxeter group. It is known that $G \subset \operatorname{Isom}\left(\mathbb{X}^{n}\right)$ is a discrete group with fundamental domain $P$. If $P$ is compact (or of finite volume), the group $G$ is called cocompact (or cofinite). Notice that a compact acute-angled polytope $P$ in $\mathbb{X}^{n}$ is simple, that is, each $k$-dimensional face of $P$ is contained in precisely $n-k$ bounding hyperplanes of $P$ (see Vinberg [21, Section 3]). In particular, a vertex of a simple Coxeter polytope is contained in exactly $n-1$ bounding hyperplanes of $P$. Denote by $\mathfrak{f}_{k}, k=0, \ldots, n-1$, the number of $k$-dimensional faces of $P$. By the Euler-Schläfli identity, one has

$$
\sum_{k=0}^{n-1}(-1)^{k} \mathfrak{f}_{k}=1-(-1)^{n} .
$$

If $P$ is simple, then obviously $n \mathfrak{f}_{0}=2 \mathfrak{f}_{1}$. We shall be mainly interested in noncocompact but cofinite hyperbolic Coxeter groups in $\operatorname{Isom}\left(\mathbb{H}^{3}\right)$. In this particular 
case, the vertices of the associated Coxeter polyhedron $P$ are $k$-valent (intersections of exactly $k$ edges of $P$ ) for $k=3$ or $k=4$, only (see Vinberg [21, Section 3]). In particular, a compact or a simple Coxeter polyhedron $P \subset \mathbb{H}^{3}$ satisfies, by (2-1), $\mathfrak{f}_{0}-\mathfrak{f}_{1}+\mathfrak{f}_{2}=2$ and $3 \mathfrak{f}_{0}=2 \mathfrak{f}_{1}$ so that the number $\mathfrak{f}_{0}=2\left(\mathfrak{f}_{2}-2\right) \geq 4$ of vertices is even, and the number $\mathfrak{f}_{1}$ of edges and dihedral angles of $P$ satisfies $\mathfrak{f}_{1}=3\left(\mathfrak{f}_{2}-2\right)$.

Consider a geometric Coxeter group with fundamental polytope $P \subset \mathbb{X}^{n}$. Denote by $S=\left\{s_{i} \mid i \in I\right\}$ the set of generating reflections of $G$. Together with the set $R$ of relations

$$
s_{i}^{2}=1, \quad\left(s_{i} s_{j}\right)^{k_{i j}}=1 \quad \text { if } \quad \angle\left(H_{i}, H_{j}\right)=\frac{\pi}{k_{i j}},
$$

we obtain the presentation $\langle S \mid R\rangle$ for $G$. The stabiliser of any vertex of $P$ is generated by the reflections in the hyperplanes passing through it and gives rise to a subgroup of $G$ which itself is a geometric Coxeter group $G_{T}$ for some $T \subset S$.

For simple presentations we prefer a description of $G$ (and of its subgroups) by means of Coxeter diagrams. More precisely, the Coxeter diagram $\Sigma=\Sigma(G)=\Sigma(P)$ of a geometric Coxeter group $G$ (and its fundamental Coxeter polytope $P$ ) consists of nodes $v_{i}, i \in I$, corresponding to the reflections $s_{i}$ (and its mirrors $H_{i}$ ), which are pairwise connected by a weighted edge $\varepsilon_{i j}=v_{i} v_{j}$ if the hyperplanes $H_{i}, H_{j}$ are not orthogonal. For hyperplanes forming the dihedral angle $\angle\left(H_{i}, H_{j}\right)=\pi / 3$, the edge $\varepsilon_{i j}$ is drawn without weight, while for hyperplanes intersecting with dihedral angle $\pi / k, k \geq 4$, the edge is marked by $k$. The nodes in $\Sigma$ corresponding to parallel hyperplanes are connected by an edge with weight $\infty$, while ultra-parallel hyperbolic hyperplanes give rise to nodes joined by a dotted edge (omitting the weight given by their hyperbolic distance). The order and the rank of the diagram $\Sigma=\Sigma(P)$ are defined by the cardinality of $S$ and by the rank of the Gram matrix of $P$. Furthermore, $\Sigma$ is called elliptic, parabolic or hyperbolic if the Coxeter group (and the Coxeter polytope $P$ ) is spherical, euclidean or hyperbolic. Hence, by Vinberg's criterion (see Section 2.1), a finite (resp. ideal) vertex of a Coxeter polytope $P \subset \mathbb{H}^{n}$ gives rise to an elliptic Coxeter diagram of rank $n$ (resp. parabolic Coxeter diagram of rank $n-1$ ) which can be identified with a certain subdiagram of $\Sigma$ (see Vinberg [22, Part II, Chapter 5]). All connected elliptic and parabolic Coxeter diagrams, and therefore all irreducible spherical and euclidean Coxeter groups, were classified by Coxeter [2] in 1934. In Table 1, we reproduce his results for order two and three, only. Furthermore, for the elliptic diagrams, we add the associated exponents (see Coxeter and Moser [3, Section 9.7]).

For the description of geometric Coxeter groups given by linear diagrams

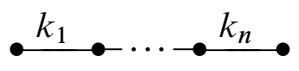




\begin{tabular}{|l|c|c|c|c|}
\hline \multicolumn{2}{|l|}{ Elliptic case } & \multicolumn{2}{l|}{ Parabolic case } \\
\hline Diagram & Notation & Exponents & Diagram & Notation \\
\hline$\bullet$ & $I_{k}$ & $1, k-1$ & $\tilde{A}_{1}$ \\
\hdashline & $A_{3}$ & $1,2,3$ & $\tilde{A}_{2}$ \\
\hdashline & $B_{3}$ & $1,3,5$ & $\widetilde{B}_{2}$ \\
\\
\hdashline
\end{tabular}

Table 1: Connected elliptic and parabolic Coxeter diagrams of orders 2 and 3

we use the Coxeter symbol $\left(k_{1}, \ldots, k_{n}\right)$. Diagrams of type (2-3) describe so-called Coxeter orthoschemes and play an important role in the theory of regular polytopes and tesselations (see Vinberg [22, Part II, Chapter 5, Section 3]). We are particularly interested in the hyperbolic Coxeter orthoscheme $(3,3,6)$ in $\mathbb{H}^{3}$, having precisely one ideal vertex related to the group $\tilde{I}=(3,6)$, and which forms a building block for an ideal regular tetrahedron. Let us provide some further instructive examples.

Example 1 The Coxeter diagram

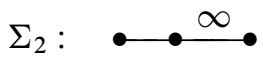

is intimately related to the modular group $\operatorname{SL}(2, \mathbb{Z})$. By a result of CL Siegel (see Floyd [4], for example), it provides the unique non-compact hyperbolic 2-orbifold $Q_{2}=\mathbb{H}^{2} / \Sigma_{2}$ of minimal volume.

Example 2 The Coxeter graph

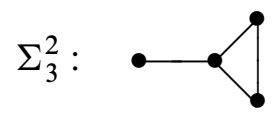

describes a hyperbolic tetrahedron with precisely one ideal and three finite vertices. As indicated by the two-fold graph symmetry in (2-5), the tetrahedron has an internal symmetry plane along which it can be dissected into two isometric copies of the Coxeter orthoscheme $\Sigma_{3}:=(3,3,6)$. Hence, the tetrahedron $\Sigma_{3}^{2}$ is the double with twice the volume of $\Sigma_{3}$. Let us point out that the quotient space $\mathbb{H}^{3} /(3,3,6)$ is distinguished by the fact that it has minimal volume among all cusped hyperbolic 3-orbifolds. This is a result of Meyerhoff [15]. 
Example 3 Let $p, q, r \geq 3$ be integers such that $1 / p+1 / q<1 / 2 \leq 1 / q+1 / r$, and consider the Coxeter diagram of order five

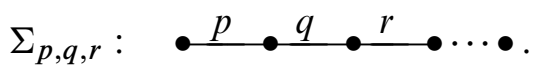

By Vinberg's criterion, one sees that the graph $\Sigma_{p, q, r}$ describes a right triangular hyperbolic Coxeter prism of finite volume, that is, a prism with one triangular face $F_{1}$ being orthogonal to the three quadrilateral faces. The prism has five finite vertices and, according to the (in-)equality $1 / q+1 / r \geq 1 / 2$, one further (finite or) ideal vertex. The infinite sequence (2-6) is related to infinite-volume hyperbolic Coxeter orthoschemes $(p, q, r)$ having one ultra-ideal vertex $v_{*}$ (with positive norm and with triangular vertex figure $(p, q))$. By cutting off from $(p, q, r)$ the part of infinite volume by means of the associated polar plane $H_{*}=\left\{x \in \mathbb{H}^{3} \mid\left\langle x, v_{*}\right\rangle=0\right\}$, we get a finite-volume triangular right Coxeter prism in $\mathbb{H}^{3}$, also called a simply truncated Coxeter 3 -orthoscheme. In the limiting case

$$
\Sigma_{\infty, q, r}: \bullet \infty \cdot q \cdot r \cdot \bullet^{\infty} \bullet, \quad \frac{1}{q}+\frac{1}{r} \geq \frac{1}{2},
$$

the polar plane $H_{*}$ is parallel to the second triangular face $F_{2}$. The polyhedron (2-7) has the combinatorial type of a pyramid over a product of two segments and has exactly one 4-valent vertex. Hence, the polyhedron $\Sigma_{\infty, q, r}$ is not simple.

In contrast to the spherical and euclidean cases, the classification of cofinite hyperbolic Coxeter groups and Coxeter polytopes is not available and far out of reach. For some families of given simple combinatorial type, there are complete classification results. For example, non-compact Coxeter simplices were classified by Koszul [14], and straight Coxeter prisms of finite volume were classified by Kaplinskaja [8]. Noncompact Coxeter simplices of finite volume exist up to dimension nine. For their volumes, we refer to Johnson, Kellerhals, Ratcliffe and Tschantz [7, page 347-348]. The 23 examples in dimension three are listed in Table 2.

\section{Growth rates of cofinite hyperbolic Coxeter groups}

\subsection{Growth functions and growth rates}

Let $G$ be a geometric Coxeter group with set $S$ of natural generators, and denote by $P \subset \mathbb{X}^{n}$ a Coxeter fundamental domain for $G$. The (spherical) growth series of $(G, S)$

$$
f_{S}(x)=1+|S| x+\sum_{k \geq 2} a_{k} x^{k},
$$



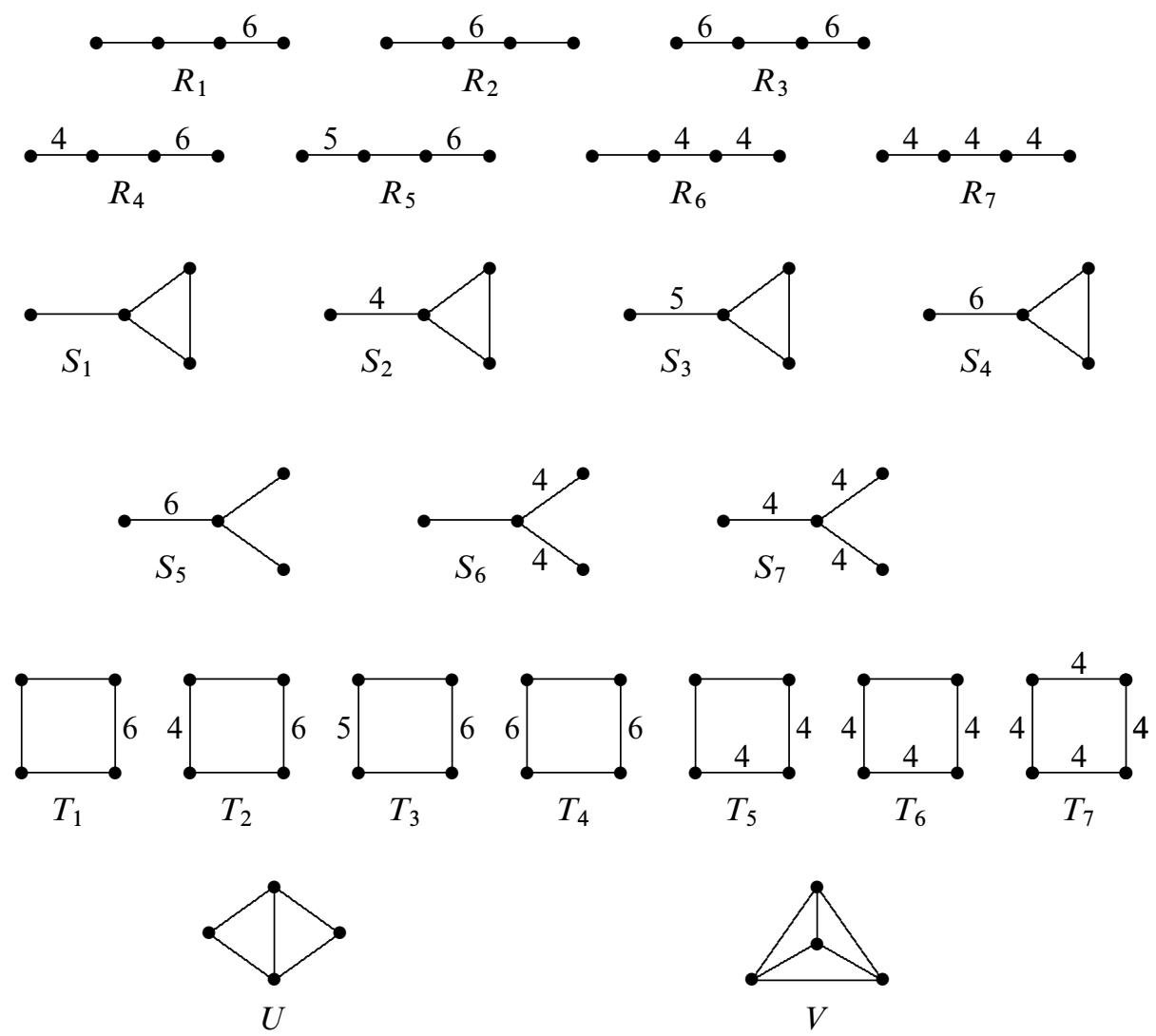

Table 2: The 23 non-compact hyperbolic Coxeter tetrahedra of finite volume

where $a_{k}$ is the number of words in $G$ of $S$-length $k$, is by Steinberg's result [20] the series expansion of a rational function, that is,

$$
f_{S}(x)=\frac{p(x)}{q(x)}, \quad \text { where } \quad p, q \in \mathbb{Z}[x]
$$

are coprime polynomials. In order to investigate growth functions, Steinberg's formula [20]

$$
\frac{1}{f_{S}\left(x^{-1}\right)}=\sum_{\substack{G_{T}<G \\\left|G_{T}\right|<\infty}} \frac{(-1)^{|T|}}{f_{T}(x)}
$$

is very important since it allows us to compute the growth function of a given group in terms of the growth functions of its finite subgroups $G_{T}$. Each such $G_{T}$ is notabene 
a spherical Coxeter group acting as stabiliser of a certain face of $P$ and yields a polynomial term $f_{T}(x)$ in (3-3). In the case of a reducible group $\left(G_{T}, T\right)=\left(G_{T_{1}} \times\right.$ $\left.G_{T_{2}}, T_{1} \cup T_{2}\right)$, the polynomial $f_{T}(x)$ equals the product of the growth polynomials of $\left(G_{T_{1}}, T_{1}\right)$ and $\left(G_{T_{2}}, T_{2}\right)$. By a result of Solomon [19], the function $f_{T}(x)$ can be derived easily in terms of its Coxeter exponents $m_{1}=1, m_{2}, \ldots, m_{t}$ (see Coxeter and Moser [3]) according to

$$
f_{T}(x)=\prod_{i=1}^{t}\left[m_{i}+1\right],
$$

where each factor in (3-4) is a polynomial of type $[k]=1+x+\cdots+x^{k-1}$. Later, we shall write $[k, l]=[k] \cdot[l]$ and so on.

\begin{tabular}{|l|l|l|l|}
\hline Notation & Symbol & $m_{1}, m_{2}, m_{3}$ & $f_{S}(x)$ \\
\hline$A_{1}$ & - & 1 & {$[2]$} \\
$D_{2}^{k}, k \geq 2$ & $(k)$ & $1, k-1$ & {$[2, k]$} \\
$A_{3}$ & $(3,3)$ & $1,2,3$ & {$[2,3,4]$} \\
$B_{3}$ & $(4,3)$ & $1,3,5$ & {$[2,4,6]$} \\
$H_{3}$ & $(5,3)$ & $1,5,9$ & {$[2,6,10]$} \\
\hline
\end{tabular}

Table 3: Growth of irreducible finite Coxeter groups of rank at most three

Since

$$
[k](x)=x^{k-1} \cdot[k]\left(x^{-1}\right)
$$

for each positive integer $k$, the polynomial $f=f_{T}$ (of degree $d$, say) in (3-3) is not only monic but also palindromic, that is, $f(x)=x^{d} f\left(x^{-1}\right)$. This property and formulas (3-2), (3-3) imply that $p(0)=1$, which together with $f_{S}(0)=1$ (see (3-1)) yields $q(0)=1$.

In the cofinite hyperbolic case, the radius of convergence $R$ of the infinite series $f_{S}(x)$ is smaller than 1 (see de la Harpe [5]), and its inverse

$$
\tau:=\limsup _{k \rightarrow \infty} \sqrt[k]{a_{k}}>1
$$

is called the growth rate of $(G, S)$ (and of $P$ ). By (3-2), $R$ is equal to the smallest real positive root of $q(x)$, and by (3-3) and (3-4), the growth rate $\tau=1 / R>1$ is an algebraic integer. 
If $G$ acts cocompactly on $\mathbb{H}^{3}$, then the growth function $f(x):=f_{S}(x)$ is antireciprocal, that is, $f\left(x^{-1}\right)=-f(x)$ (see Charney and Davis [1]). Since $p(x)$ is palindromic by (3-3) and (3-4), the denominator $q(x)$ (of degree $d$, say) is antipalindromic, that is, $q(x)=-x^{d} q\left(x^{-1}\right)$. Therefore, $\tau$ and $1 / \tau$ are Galois conjugates.

In the non-cocompact case, the anti-reciprocity property does not hold anymore. As an illustration, consider the non-cocompact cofinite Coxeter groups $G_{1}=R_{1}=(3,3,6)$, $G_{2}=V$, which is related to an ideal regular tetrahedron, and $G_{3}=\Sigma_{\infty, 3,3}$ (see Table 2 and (2-7)). By (3-3) and Table 3, and by using some well-known factorisation properties of $[k]$ such as $[2 k]=\left(x^{k}+1\right)[k]$, their growth functions $f_{1}, f_{2}$ and $f_{3}$ can be computed as follows (see also Komori and Umemoto [13, Proposition 1]).

$$
\begin{aligned}
& f_{1}(x)=\frac{[2,2,2,3]\left(x^{2}+1\right)\left(x^{2}-x+1\right)}{(x-1)\left(x^{7}+x^{6}+x^{5}+x^{4}-1\right)}, \\
& f_{2}(x)=\frac{[2,3]}{(x-1)\left(3 x^{2}+x-1\right)}, \\
& f_{3}(x)=\frac{[2,2,2,3]\left(x^{2}+1\right)}{(x-1)\left(x^{5}+2 x^{4}+2 x^{3}+x^{2}-1\right)} .
\end{aligned}
$$

The fact that $x=1$ is a pole of $f_{i}$ follows from the vanishing of the Euler characteristic $\chi\left(G_{i}\right)$. For the numerators $p_{i}$ and denominators $q_{i}$ of $f_{i}(i=1,2,3)$ in (3-7), we see that $\operatorname{deg} p_{1} \neq \operatorname{deg} q_{1}, q_{2}$ is not monic, and we calculate

$$
\begin{aligned}
x^{9} q_{1}\left(x^{-1}\right) & =x^{9}-x^{8}-x^{5}+x \\
& =x(x-1)\left(x^{7}-x^{3}-x^{2}-x-1\right), \\
x^{3} q_{2}\left(x^{-1}\right) & =x^{3}-2 x^{2}-2 x+3 \\
& =(x-1)\left(x^{2}-x-3\right), \\
x^{6} q_{3}\left(x^{-1}\right) & =x^{6}-x^{5}-x^{4}-x^{3}+x+1 \\
& =(x-1)\left(x^{5}-x^{3}-2 x^{2}-2 x-1\right) .
\end{aligned}
$$

As a consequence, the functions $f_{i}$ are not anti-reciprocal. The growth rates are given by

$$
\tau((3,3,6)) \simeq 1.296466, \tau(V) \simeq 2.302776, \tau\left(\Sigma_{\infty, 3,3}\right) \simeq 1.734691
$$

Finally, one can check numerically that the Galois conjugates of $\tau((3,3,6))$ lie inside and outside of the unit circle but are all of absolute value strictly smaller than $\tau((3,3,6))$. The Galois conjugates of $\tau\left(\Sigma_{\infty, 3,3}\right)$ lie all inside the unit circle. 


\subsection{Pisot numbers and Perron numbers}

A very interesting arithmetic aspect in the study of growth rates of cofinite hyperbolic Coxeter groups is that certain classes of real algebraic integers show up. An algebraic integer $\sigma>1$ is a Salem number if its inverse $1 / \sigma$ is a Galois conjugate of $\sigma$ and all other Galois conjugates lie on the unit circle. It is known by results of Cannon, Wagreich and Parry (see Parry [16], for example) that the growth rate of a Coxeter group acting cocompactly on $\mathbb{H}^{2}$ or $\mathbb{H}^{3}$ is a Salem number. In [9], the author and Kolpakov showed that the cocompact hyperbolic Coxeter group of minimal growth rate in three dimensions is the tetrahedral group $(3,5,3)$, while E Hironaka proved in [6] that the triangle group $(3,7)$ is the cocompact hyperbolic Coxeter group of minimal growth rate in two dimensions. Both Coxeter groups are closely related to the (unique) compact hyperbolic orbifolds of minimal volume in dimensions two and three (see [9], for example).

An algebraic integer $\alpha>1$ is a Pisot-Vijayaraghavan number, or a Pisot number for short, if all its Galois conjugates are less than 1 in absolute value. In contrast to Salem numbers, the smallest Pisot number is known. More precisely, Smyth [18] proved that this one is given by the algebraic integer $\alpha_{S} \simeq 1.324718$ with minimal polynomial $x^{3}-x-1$. Floyd [4] proved that the growth rate of any non-cocompact cofinite planar hyperbolic Coxeter group is a Pisot number and then, based on Smyth's result, that the smallest growth rate equals $\alpha_{S}$ and is realised by the triangle group $(3, \infty)$.

Finally, an algebraic integer $\beta>1$ is called a Perron number if all its Galois conjugates are less than $\beta$ in absolute value. Of course, any Pisot or Salem number is a Perron number.

In [12], Komori and Umemoto show among other things that the growth rates of the non-cocompact groups $G_{i}, i=1,2,3$, in Section 3.1 and of the Coxeter tetrahedra in Table 2 are all Perron numbers. However, neither $\tau((3,3,6))$ nor $\tau(V)$ are Pisot number. While this is evident for $\tau(V)$, having minimal polynomial $x^{2}-x-3$, the verification for $\tau((3,3,6))$, with minimal polynomial $x^{7}-x^{3}-x^{2}-x-1$, follows by comparing $\tau((3,3,6))<\alpha_{S}$ and by using Smyth's minimality result mentioned above (see (3-8)). In contrast to this, a numerical check shows that $\tau\left(\Sigma_{\infty, 3,3}\right)$ is a Pisot number with minimal polynomial $x^{5}-x^{3}-2 x^{2}-2 x-1$. This can be shown rigorously as follows (see Example 7 below).

In Kolpakov [11], a geometric characterisation of Pisot numbers has been proven which explains to some extent the above discrepancies. Consider a Coxeter polyhedron $P \subset \mathbb{H}^{3}$ of finite volume. An edge $e$ of $P$ is a ridge of type $\langle 2,2, n, 2,2\rangle$ if $e$ is bounded with 3 -valent vertices $v, w$ such that the dihedral angles at the incident edges 
equal $\pi / 2$ while the dihedral angle at the edge $e$ equals $\pi / n$. If a Coxeter polyhedron $P_{\infty}$ has a 4-valent ideal vertex, then Vinberg [22, page 238] indicated the following degeneration feature which was proved in detail by Kolpakov [11, Proposition 2].

Proposition 4 Let $P_{\infty} \subset \mathbb{H}^{3}$ be a Coxeter polyhedron of finite volume with at least one 4-valent ideal vertex $v_{\infty}$. Then there exists a sequence of finite-volume Coxeter polyhedra $P_{n} \subset \mathbb{H}^{3}$ having the same combinatorial type and dihedral angles as $P_{\infty}$ except for a ridge $e$ of type $\langle 2,2, n, 2,2\rangle$ with $n$ sufficiently large, giving rise to the vertex $v_{\infty}$ under contraction of $e$ as $n \rightarrow \infty$.

Based on this point of view, Kolpakov [11, Proposition 3 and Theorem 5] proved the following results, which generalise Floyd's work [4] from the planar to the spatial case.

Proposition 5 Let $P_{\infty} \subset \mathbb{H}^{3}$ be a Coxeter polyhedron of finite volume with at least one 4-valent ideal vertex obtained from a sequence of finite-volume Coxeter polyhedra $P_{n}$ by contraction of a ridge of type $\langle 2,2, n, 2,2\rangle$ as $n \rightarrow \infty$. Then, the growth rates $\tau\left(P_{n}\right)$ tend from below to the growth rate $\tau\left(P_{\infty}\right)$.

Proposition 6 Let $P_{n} \subset \mathbb{H}^{3}$ be a compact Coxeter polyhedron with a ridge $e$ of type $\langle 2,2, n, 2,2\rangle$ for sufficiently large $n$. Denote by $P_{\infty}$ the polyhedron arising by contraction of the ridge $e$. Let $\tau_{n}$ and $\tau_{\infty}$ be the growth rates of $P_{n}$ and $P_{\infty}$, respectively. Then $\tau_{n}<\tau_{\infty}$ for all $n$, and $\tau_{n} \rightarrow \tau_{\infty}$ as $n \rightarrow \infty$. Furthermore, $\tau_{\infty}$ is a Pisot number.

Example 7 Consider the sequence $\Sigma_{p, 3,3}, p \geq 7$, of cocompact hyperbolic Coxeter groups (see (2-6)). Each member $\Sigma_{p, 3,3}$ is a simple straight triangular Coxeter prism, that is, a compact simply truncated Coxeter orthoscheme. The dihedral angle $\pi / p$ sits at an edge $e$, which connects the top and bottom faces $F_{1}$ and $F_{2}$, and which is of type $\langle 2,2, p, 2,2\rangle$. For $p \rightarrow \infty$, the sequence $\Sigma_{p, 3,3}$ degenerates under contraction of $e$ to the polyhedron $\Sigma_{\infty, 3,3}$ with precisely one 4-valent ideal vertex $v_{\infty}$, whose stabiliser in the group

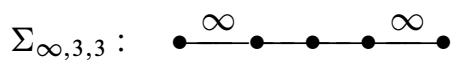

is the quadrilateral affine Coxeter group $\widetilde{A}_{1} \times \tilde{A}_{1}$. Now, by Proposition 6 , the growth rates $\tau\left(\Sigma_{p, 3,3}\right), p \geq 7$, which are all Salem numbers, tend from below to the Pisot number $\tau\left(\Sigma_{\infty, 3,3}\right) \simeq 1.734691$. 


\section{The main result}

Let $G$ be a cofinite hyperbolic Coxeter group acting on hyperbolic 3-space with noncompact fundamental Coxeter polyhedron $P \subset \mathbb{H}^{3}$. Denote by $\Omega_{0}=\Omega_{f} \cup \Omega_{\infty}$ the set of vertices of $P$ where $\Omega_{f}$ and $\Omega_{\infty} \neq \varnothing$ denote the subsets of finite vertices and ideal vertices of $P$. In the case of the Coxeter orthoscheme $(3,3,6)$, the set $\Omega_{0}$ consists of four 3-valent vertices, and $\left|\Omega_{\infty}\right|=1$.

Theorem Among all hyperbolic Coxeter groups with non-compact fundamental polyhedron of finite volume in $\mathbb{H}^{3}$, the tetrahedral group $(3,3,6)$ has minimal growth rate, and as such the group is unique.

Before we provide a proof of the Theorem, let us recapitulate the growth data of $(3,3,6)$ (see Section 3). The growth function of $(3,3,6)$ is given by (see $(3-7))$

$$
f_{(3,3,6)}(x)=\frac{[2,2,2,3]\left(x^{2}+1\right)\left(x^{2}-x+1\right)}{(x-1)\left(x^{7}+x^{6}+x^{5}+x^{4}-1\right)},
$$

and the growth rate $\tau_{(3,3,6)} \simeq 1.296466$ has minimal polynomial $x^{7}-x^{3}-x^{2}-x-1$ (see (3-8)). As already mentioned in Section 3.2, it is known that $\tau_{(3,3,6)}$ is a Perron number, but it is not a Pisot number since its value is strictly smaller than the least Pisot number $\alpha_{S} \simeq 1.324718$.

\subsection{Proof of the Theorem}

Consider a hyperbolic Coxeter group $G=(G, S)$ with non-compact fundamental Coxeter polyhedron $P \subset \mathbb{H}^{3}$ of finite volume. The polyhedron $P$ is the convex hull of finitely many points in $\mathbb{H}^{3} \cup \partial \mathbb{H}^{3}$, whose number is denoted by $\mathfrak{f}_{0}$ as usually (see Section 2.2). Together with the number $\mathfrak{f}_{1}$ of edges and the number $\mathfrak{f}_{2}$ of polygonal faces of $P$, we have that $\mathfrak{f}_{0}-\mathfrak{f}_{1}+\mathfrak{f}_{2}=2$. We shall focus on the non-empty vertex subset $\Omega_{\infty} \subset \Omega_{0}$ of $P$ and the valencies of its elements. For $i=3,4$, we denote by $\Omega_{\infty}^{i}$ the set of $i$-valent ideal vertices of $P$ and recall that $\Omega_{\infty}=\Omega_{\infty}^{3} \cup \Omega_{\infty}^{4}$.

Step 1 Suppose that $\Omega_{\infty}^{4} \neq \varnothing$ and let $v_{\infty} \in \Omega_{\infty}^{4}$. By Proposition 4, $P$ is the result of a contraction process by means of finite-volume Coxeter polyhedra $P_{n} \subset \mathbb{H}^{3}$ having the same combinatorial type and dihedral angles as $P$ except for a ridge of type $\langle 2,2, n, 2,2\rangle$ with $n$ sufficiently large, giving rise to the vertex $v_{\infty}$. By Proposition 5, the growth rates $\tau\left(P_{n}\right)$ tend from below to $\tau(P)$.

If $\left|\Omega_{\infty}\right|=1$, then the polyhedra $P_{n}$ are all compact, and by Proposition $6, \tau(P)$ is a Pisot number. Since $\tau_{(3,3,6)}$ is smaller than any Pisot number, the conclusion follows. 
If $\left|\Omega_{\infty}\right| \geq\left|\Omega_{\infty}^{4}\right| \geq 2$, we perform the contraction process successively for each further vertex in $\Omega_{\infty}^{4}$, by using Proposition 4 , so that Proposition 5 allows us to conclude that non-compact Coxeter polyhedra in $\mathbb{H}^{3}$ of smallest growth rates are characterised by $\Omega_{\infty}^{4}=\varnothing$.

Step 2 Let $\Omega_{\infty}^{4}=\varnothing$, that is, $\Omega_{0}=\Omega_{f} \cup \Omega_{\infty}^{3}$. In particular, all vertices of $P$ are 3 -valent and

$$
\mathfrak{f}_{0}=2\left(\mathfrak{f}_{2}-2\right) \geq 4, \quad \mathfrak{f}_{1}=\frac{3}{2} \mathfrak{f}_{0} .
$$

Denote by $\pi / n_{i}$ for integers $n_{i} \geq 2, i=1, \ldots, \mathfrak{f}_{1}$, the dihedral angles of $P$. By Steinberg's formula (see (3-3)) and Table 3,

$$
\frac{1}{f_{S}\left(x^{-1}\right)}=\sum_{\substack{G_{T}<G \\\left|G_{T}\right|<\infty}} \frac{(-1)^{|T|}}{f_{T}(x)}=1-\frac{|S|}{[2]}+\sum_{i=1}^{f_{1}} \frac{1}{\left[2, n_{i}\right]}-\sum_{v \in \Omega_{f}} \frac{1}{f_{v}(x)},
$$

where $f_{v}$ is the growth polynomial of the finite Coxeter group $G_{v}$ of rank three which is the stabiliser of the vertex $v \in \Omega_{f}$ in $G$. In Table 3 are listed all possible irreducible components for realisations of $G_{v}$. By Solomon's formula (3-4), the growth polynomial $f_{v}$ equals $\left[2, m_{2}+1, m_{3}+1\right]$ where $m_{2}, m_{3}$ depend on $G_{v}$ according to Table 3 .

We point out the following simple, but crucial fact which we term "exponent monotonicity". Let $G_{1} \neq A_{1}$ be a group in Table 3. By increasing one entry in the Coxeter symbol of $G_{1}$ and passing from $G_{1}$ to another group $G_{2}$ in Table 3, the exponents different from $m_{1}=1$ all increase as well. For example, the passage from $B_{3}$ to $H_{3}$ (increase the first entry 4 of the Coxeter symbol $(4,3)$ to 5$)$ transforms the non-trivial exponents according to $m_{2}: 3 \mapsto 5$ and $m_{3}: 5 \mapsto 9$.

Now, since $|S|=\mathfrak{f}_{2}=\mathfrak{f}_{0} / 2+2$, and since each of the $\mathfrak{f}_{1}$ edges has precisely two vertices, (4.3) can be rewritten as

$$
\begin{aligned}
\frac{1}{f_{S}\left(x^{-1}\right)} & =1-\frac{\mathfrak{f}_{2}}{[2]}+\frac{1}{2} \sum_{w \in \Omega_{0}} \sum_{i=1}^{3} \frac{1}{\left[2, n_{i}^{w}\right]}-\sum_{v \in \Omega_{f}} \frac{1}{\left[2, m_{2}+1, m_{3}+1\right]} \\
& =\frac{1}{[2]}\left\{x-1+\frac{1}{2} \sum_{w \in \Omega_{0}}\left(\sum_{i=1}^{3} \frac{1}{\left[n_{i}^{w}\right]}-1\right)-\sum_{v \in \Omega_{f}} \frac{1}{\left[m_{2}+1, m_{3}+1\right]}\right\},
\end{aligned}
$$

where we denote by $\pi / n_{i}^{w}, i=1,2,3$, the dihedral angles at the three edges giving rise to the vertex $w \in \Omega_{0}$. By definition of $[n]$, we have

$$
x^{n}-1=(x-1)[n],
$$


which, together with the working hypothesis $\Omega_{0}=\Omega_{\infty}^{3} \cup \Omega_{f}$, allows us to write

$$
\begin{aligned}
\frac{1}{f_{S}\left(x^{-1}\right)} & =\frac{x-1}{[2]}+\frac{x-1}{2[2]} \sum_{w \in \Omega_{\infty}^{3}}\left(\sum_{i=1}^{3} \frac{1}{x^{n_{i}^{w}}-1}-\frac{1}{x-1}\right)+ \\
& +\frac{x-1}{2[2]} \sum_{v \in \Omega_{f}}\left(\sum_{i=1}^{3} \frac{1}{x^{n_{i}^{v}}-1}-\frac{1}{x-1}-\frac{2(x-1)}{\left(x^{m_{2}+1}-1\right)\left(x^{m_{3}+1}-1\right)}\right) .
\end{aligned}
$$

By analysing the different types of finite subgroups $G_{v}$ according to Table 3, Parry identified the terms in the sum running over the vertices $v \in \Omega_{f}$ in the following coherent way (see [16, (2.13) and (2.14)]).

$$
\begin{array}{r}
\frac{x-1}{2[2]} \sum_{v \in \Omega_{f}}\left(\sum_{i=1}^{3} \frac{1}{x^{n_{i}^{v}}-1}-\frac{1}{x-1}-\frac{2(x-1)}{\left(x^{m_{2}+1}-1\right)\left(x^{m_{3}+1}-1\right)}\right) \\
=-\frac{1}{2} x(x-1) \sum_{v \in \Omega_{f}} \frac{\left(x^{m_{1}}-1\right)\left(x^{m_{2}}-1\right)\left(x^{m_{3}}-1\right)}{\left(x^{m_{1}+1}-1\right)\left(x^{m_{2}+1}-1\right)\left(x^{m_{3}+1}-1\right)}
\end{array}
$$

where we used $m_{1}=1$. As for the sum running over the infinite vertices $w \in \Omega_{\infty}^{3}$ in (4-6), Table 1 shows that each term belongs to a euclidean subgroup $G_{w}$ of type $\tilde{A}_{2}$, $\widetilde{B}_{2}$ or $\widetilde{I}$ (cf. also Section 2.1). An easy calculation in each of these cases reveals that

$$
\sum_{w \in \Omega_{\infty}^{3}}\left(\sum_{i=1}^{3} \frac{1}{x^{n_{i}^{w}}-1}-\frac{1}{x-1}\right)=\frac{-\left(\left[n_{w}-1\right]+1\right)}{\left[n_{w}\right]}
$$

where

$$
n_{w}:=\max \left(n_{1}^{w}, n_{2}^{w}, n_{3}^{w}\right)=\left\{\begin{array}{lll}
3 & \text { if } & G_{w}=\widetilde{A}_{2} \\
4 & \text { if } & G_{w}=\widetilde{B}_{2} \\
6 & \text { if } & G_{w}=\widetilde{I}
\end{array}\right.
$$

By (4-5) and (4-7)-(4-9), the identity (4-6) can be rewritten according to

$$
\frac{1}{f_{S}\left(x^{-1}\right)}=\frac{x-1}{x+1}\left\{1-\frac{1}{2}\left(\sum_{w \in \Omega_{\infty}^{3}} \frac{\left[n_{w}-1\right]+1}{\left[n_{w}\right]}+x \sum_{v \in \Omega_{f}} \frac{\left[m_{2}, m_{3}\right]}{\left[m_{2}+1, m_{3}+1\right]}\right)\right\}
$$

Now, the inversion $x \mapsto x^{-1}$ for $x \neq 0$ combined with (3-5), that is,

$$
\frac{1}{[n]\left(x^{-1}\right)}=\frac{x^{n-1}}{[n](x)}=\frac{x^{n-1}}{[n]}
$$


transforms (4-10) into the expression

$$
\begin{aligned}
\frac{1}{f_{S}(x)} & =\frac{1-x}{1+x}\left\{1-\frac{x}{2}\left(\sum_{w \in \Omega_{\infty}^{3}} \frac{\left[n_{w}-1\right]+x^{n_{w}-2}}{\left[n_{w}\right]}+\sum_{v \in \Omega_{f}} \frac{\left[m_{2}, m_{3}\right]}{\left[m_{2}+1, m_{3}+1\right]}\right)\right\} \\
& =: \frac{1-x}{[2]}\left\{1-\frac{x}{2} H(x)\right\},
\end{aligned}
$$

where

$$
H(x):=\sum_{w \in \Omega_{\infty}^{3}} \frac{\left[n_{w}-1\right]+x^{n_{w}-2}}{\left[n_{w}\right]}+\sum_{v \in \Omega_{f}} \frac{\left[m_{2}, m_{3}\right]}{\left[m_{2}+1, m_{3}+1\right]} .
$$

In order to prove the Theorem it suffices to show that, for each $(G, S)$ different from $(3,3,6)$, and for all $x \in\left(0,1 / \tau_{(3,3,6)}\right]$,

$$
\frac{1}{f_{S}(x)}<\frac{1}{f_{(3,3,6)}(x)}
$$

which, by (4-9), (4-11) and (4-12), is equivalent to (see also (4-1))

$$
\begin{aligned}
H(x)>H_{(3,3,6)}(x) & =\frac{[5]+x^{4}}{[6]}+\frac{[5]}{[2,6]}+\frac{[2]}{[2,3]}+\frac{[2,3]}{[3,4]} \\
& =2 \frac{1+[2]\left(x^{6}+2 x^{5}+2 x^{4}+3 x^{3}+2 x^{2}+2 x+1\right)}{[2,2,3]\left(x^{2}+1\right)\left(x^{2}-x+1\right)} .
\end{aligned}
$$

To this end, we consider the function in (4-12) and write

$$
H(x)=\sum_{w \in \Omega_{\infty}^{3}} g_{n_{w}}(x)+\sum_{v \in \Omega_{f}} h_{m_{2}+1}(x) h_{m_{3}+1}(x),
$$

where we put

$$
h_{k}(x)=\frac{[k-1]}{[k]} \text { and } g_{k}(x)=h_{k}(x)+\frac{x^{k-2}}{[k]}=\frac{[k-1]+x^{k-2}}{[k]} .
$$

Observe that the functions $h_{k}(x)$ are strictly monotonely decreasing on [0, 1]. Furthermore, the functions $h_{k}$ and $g_{k}$ satisfy the following properties.

Lemma 8 For all integers $k \geq 2$ and for all $x \in(0,1)$,

(a) $0<h_{k}(x)<h_{k+1}(x)<1$,

(b) $g_{k}(x)>g_{k+1}(x)>1$, and

(c) $g_{6}(x)>\frac{1}{[2]^{2}}$. 
Proof Claim (a) follows from Kellerhals and Kolpakov [9, Lemma]. For the proof of (b), we observe first that the definition of $[k]$ and $g_{k}$ according to (4-16) imply that $g_{k}(x)>1$ for all $x \in(0,1)$ and all integers $k \geq 2$. Secondly, for the difference function $d_{k}(x)=g_{k}(x)-g_{k+1}(x)$, we compute

$$
d_{k}(x)=\frac{[k+1]\left([k-1]+x^{k-2}\right)-[k]\left([k]+x^{k-1}\right)}{[k, k+1]} .
$$

Since (see Kellerhals and Perren [10, (2.4)])

$$
\left[n_{1}, n_{2}\right]=\frac{\left[n_{1}+n_{2}\right]-\left[n_{1}\right]-\left[n_{2}\right]}{x-1},
$$

for all integers $n_{1}, n_{2} \geq 2$, the numerator of $d_{k}$ yields, by (4-17) and (4-18), the estimate

$$
\begin{aligned}
\frac{2[k]-[k+1]-[k-1]}{x-1}+x^{k-2}([k+1]-x[k]) & =\frac{x^{k-1}(1-x)}{x-1}+x^{k-2} \\
& =x^{k-2}(1-x)>0 .
\end{aligned}
$$

Finally, the inequality (c) is equivalent to the (obvious) positivity of the expression

$$
[2]^{2}\left([5]+x^{4}\right)-[6]=x[2]\left(2 x^{4}+2 x^{3}+2 x^{2}+x+2\right),
$$

so that, by definition (4-16), claim (c) follows.

In order to prove (4-14), the strategy is to distinguish between the two cases $\mathfrak{f}_{2}=4$ and $\mathfrak{f}_{2} \geq 5$ and to find a respective "sandwich function" $\widetilde{H}(x)$ satisfying

$$
H(x) \geq \tilde{H}(x)>H_{(3,3,6)}(x)
$$

for all $x \in\left(0,1 / \tau_{(3,3,6)}\right]$ which is easier to handle for our purpose. It will turn out that the delicate case is $\mathfrak{f}_{2}=4$ (and in particular the groups $R_{2}$ and $R_{6}$ ) requiring a certain amount of case-by-case analysis in view of Table 2 .

Case 1 Suppose that $\mathfrak{f}_{2} \geq 5$. By (4-2), this implies that $\mathfrak{f}_{0} \geq 6$, and by hypothesis, we have that $\left|\Omega_{\infty}^{3}\right| \geq 1$. By Lemma 8 ,

$$
g_{l}(x)>g_{6}(x)>1>h_{k}(x)>h_{k}(x) h_{2}(x) \geq h_{2}^{2}(x)>0
$$

for integers $k \geq 2, l=2, \ldots, 5$, and for all $x \in(0,1)$. In view of (4-14) and (4-15), this motivates the definition of

$$
\tilde{H}(x):=\frac{[5]+x^{4}}{[6]}+\frac{5}{[2]^{2}},
$$


which leads to the estimate

$$
H(x)=\sum_{w \in \Omega_{\infty}^{3}} g_{n_{w}}(x)+\sum_{v \in \Omega_{f}} h_{m_{2}+1}(x) h_{m_{3}+1}(x) \geq \widetilde{H}(x)
$$

on the interval $(0,1)$. Since

$$
\frac{5}{[2]^{2}}-\left(\frac{[5]}{[2,6]}+\frac{[2]}{[2,3]}+\frac{[2,3]}{[3,4]}\right)=\frac{(x-1)^{2}\left(2 x^{4}+3 x^{2}+2\right)}{[2,2,3]\left(x^{2}+1\right)\left(x^{2}-x+1\right)}>0,
$$

it follows from (4-20) that $\tilde{H}(x)>H_{(3,3,6)}(x)$ for all $x \in\left(0,1 / \tau_{(3,3,6)}\right.$, Hence, the conclusion (4-14) follows.

Case 2 Suppose that $\mathfrak{f}_{2}=4$, that is, $P$ is one of the 23 non-compact Coxeter tetrahedra which are listed in Table 2. Although the combinatorial type of $P$ is most elementary, the proof of $\tau(P) \geq \tau((3,3,6))$ with equality only if $P$ is isometric to $(3,3,6)$ is more delicate.

(i) Suppose first that $P$ is an ideal Coxeter tetrahedron, that is, $P=T_{4}, T_{7}$ or $V$ (cf. Table 2). Then, by (4-12), (4-14) and by Lemma 8 ,

$$
\begin{aligned}
H(x) & =\sum_{w \in \Omega_{\infty}^{3}} \frac{\left[n_{w}-1\right]+x^{n_{w}-2}}{\left[n_{w}\right]} \geq 4 \frac{[5]+x^{4}}{[6]}>\frac{[5]+x^{4}}{[6]}+3> \\
& >\frac{[5]+x^{4}}{[6]}+\frac{[5]}{[2,6]}+\frac{[2]}{[2,3]}+\frac{[2,3]}{[3,4]}=H_{(3,3,6)}(x),
\end{aligned}
$$

which holds for all $x \in(0,1)$ and finishes the verification in this particular case.

(ii) Let us treat another simple case, namely $P=U$, given by the Coxeter diagram

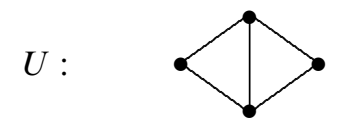

which, by Lemma 8 , yields

$$
H_{U}(x)=2 \frac{[2]+x}{[3]}+2 \frac{[2,3]}{[3,4]}>\frac{[5]+x^{4}}{[6]}+\frac{[5]}{[2,6]}+\frac{1}{[3]}+\frac{[2,3]}{[3,4]}=H_{(3,3,6)} .
$$

(iii) Consider the Coxeter groups (see Table 2)

$$
\Sigma_{k}: \bullet k \bullet \bullet 6, \quad k=3,4,5,6,
$$

where $\Sigma_{3}=(3,3,6)=R_{1}, \Sigma_{4}=R_{4}, \Sigma_{5}=R_{5}$ and $\Sigma_{6}=R_{3}$. We want to show that, for $k=4,5,6$,

$$
H_{\Sigma_{k}}(x)>H_{(3,3,6)}(x) \text { for all } x \in(0,1),
$$


by exploiting the exponent monotonicity for $m_{2}, m_{3}$. Indeed, by (4-12) and by Lemma 8, we obtain, for $k=4,5$,

$$
\begin{aligned}
H_{\Sigma_{k}}(x)-H_{(3,3,6)}(x) & =\frac{[5]+x^{4}}{[6]}+\sum_{v \in \Omega_{f}} \frac{\left[m_{2}, m_{3}\right]}{\left[m_{2}+1, m_{3}+1\right]}-H_{(3,3,6)}(x) \\
& =\frac{[k-1]}{[2, k]}-\frac{[2]}{[2,3]}+\frac{\left[m_{2}, m_{3}\right]}{\left[m_{2}+1, m_{3}+1\right]}-\frac{[2,3]}{[3,4]}>0,
\end{aligned}
$$

where the exponents $m_{2}, m_{3}$ are associated to the subgroup $(k, 3)$ of $\Sigma_{k}$. In a similar way, we can conclude that on $(0,1)$

$$
H_{\Sigma_{6}}(x)=2 \frac{[5]+x^{4}}{[6]}+\sum_{v \in \Omega_{f}} \frac{\left[m_{2}, m_{3}\right]}{\left[m_{2}+1, m_{3}+1\right]}>H_{(3,3,6)}(x) .
$$

(iv) Consider the Coxeter groups (see Table 2)

$$
T_{k}, \quad k=3,4,5:
$$

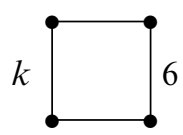

which have all $\left|\Omega_{\infty}^{3}\right|=2$. We proceed as in (iii) and see that the functions

$$
H_{T_{k}}(x)=2 \frac{[5]+x^{4}}{[6]}+2 \frac{\left[m_{2}, m_{3}\right]}{\left[m_{2}+1, m_{3}+1\right]},
$$

where the exponents $m_{2}, m_{3}$ are again associated to the subgroup $(k, 3)$ of $T_{k}$, are strictly bigger than $H_{(3,3,6)}(x)$ for $x \in(0,1)$.

(v) Let us pass to the Coxeter groups with precisely one subgroup of type $\tilde{A}_{2}$
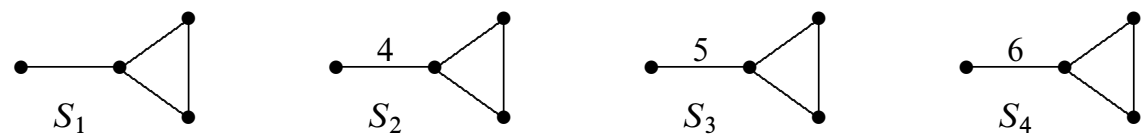

which yield - for $k=1,2,3$ - the functions

(4-25) $H_{S_{k}}(x)=\frac{[2]+x}{[3]}+\frac{1}{[2]^{2}}+2 \frac{\left[m_{2}, m_{3}\right]}{\left[m_{2}+1, m_{3}+1\right]} \geq \frac{[2]+x}{[3]}+\frac{1}{[2]^{2}}+2 \frac{[2,3]}{[3,4]}$,

where we again exploited Lemma 8 and the monotonicity properties of $m_{2}, m_{3}$. It follows from (4-14) and (4-25) that $H_{S_{k}}(x)>H_{(3,3,6)}(x)$ on $(0,1)$ if

$$
\frac{1}{[2]^{2}}+\frac{[2,3]}{[3,4]}>\frac{[5]}{[2,6]}+\frac{1}{[3]}
$$


which is equivalent to the positivity of the associated difference function $\Delta(x)$ as given by

$$
\Delta(x)=\frac{x^{2}(x-1)^{2}}{[2,2,3]\left(x^{2}+1\right)\left(x^{2}-x+1\right)} .
$$

As for the group $S_{4}$ with $\left|\Omega_{f}\right|=1$, it follows easily from (4-27) that

$$
H_{S_{4}}(x)=2 \frac{[5]+x^{4}}{[6]}+\frac{[2]+x}{[3]}+\frac{1}{[2]^{2}}>H_{(3,3,6)}(x) .
$$

(vi) Next, we treat the Coxeter groups $S_{6}, S_{7}, T_{5}$ and $T_{6}$ which have among its euclidean subgroups only those of type $\widetilde{B}_{2}$, and have, if $\left|\Omega_{\infty}^{3}\right|<3$, two irreducible spherical subgroups of rank three. By computing the respective functions $H(x)$ according to (4-12) and by using the inequality (4-26), one can easily see that $H(x)>$ $H_{(3,3,6)}(x)$ on $(0,1)$. Let us illustrate this for the Coxeter group

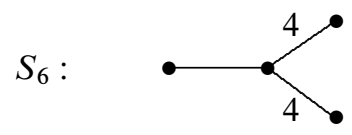

with $\left|\Omega_{\infty}^{3}\right|=1$ and whose function $H(x)$ compares with $H_{(3,3,6)}(x)$ according to (see Lemma 8, (4-14) and (4-26))

(4-29) $H(x)=\frac{[3]+x^{2}}{[4]}+2 \frac{[5,9]}{[6,10]}+\frac{1}{[2]^{2}}>\frac{[5]+x^{4}}{[6]}+2 \frac{[2,3]}{[3,4]}+\frac{1}{[2]^{2}}>H_{(3,3,6)}(x)$.

(vii) In a similar way as in (4-29), and based on Lemma 8 and (4-26) as well, we can conclude that the Coxeter group

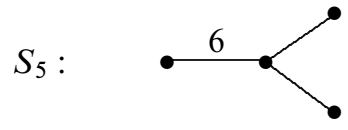

satisfies $H(x)>H_{(3,3,6)}(x)$ on $(0,1)$.

(viii) We finish the proof by considering the remaining Coxeter tetrahedra
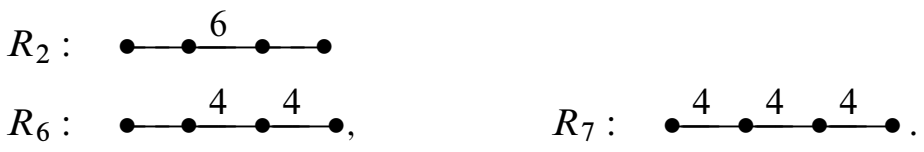

First, by Lemma 8 , it is evident that, on $(0,1)$,

(4-30) $H_{R_{7}}(x)=2 \frac{[3]+x^{2}}{[4]}+2 \frac{[3]}{[2,4]}>\frac{[3]+x^{2}}{[4]}+\frac{[5,9]}{[6,10]}+\frac{[3]}{[2,4]}+\frac{1}{[3]}=H_{R_{6}}(x)$. 
Hence, it remains to prove (4-14) for the two simply asymptotic orthoschemes $R_{2}$ and $R_{6}$. To this end, we compute the difference functions $\Delta_{i}(x):=H_{R_{i}}(x)-H_{(3,3,6)}(x)$ for $i=2,6$. By using (4-14) and $[2 k]=[k]\left(1+x^{k}\right)$, as usually, we easily deduce the expressions

$$
\Delta_{2}(x)=2 \frac{x^{3}[5]}{[2,2,3]\left(x^{2}+1\right)\left(x^{2}-x+1\right)}=x[5] \Delta_{6}(x),
$$

which are clearly positive on $(0,1)$.

\section{Final remarks}

\subsection{About the function $H_{(3,3,6)}$ and a related Salem polynomial}

Consider the extremal tetrahedral group $(3,3,6)$, providing the minimal volume cusped hyperbolic 3-orbifold and the non-cocompact Coxeter group with minimal growth rate $\tau_{*} \simeq 1.296466$. As pointed out, the minimal polynomial $x^{7}-x^{3}-x^{2}-x-1$ of $\tau_{*}$ is neither a Salem nor a Pisot polynomial. However, $\tau_{*}$ is a Perron number. Consider the auxiliary function (see (4-14))

$$
\begin{aligned}
H_{(3,3,6)}(x) & =2 \frac{1+[2]\left(x^{6}+2 x^{5}+2 x^{4}+3 x^{3}+2 x^{2}+2 x+1\right)}{[2,2,3]\left(x^{2}+1\right)\left(x^{2}-x+1\right)} \\
& =: 2 \frac{1+[2] p(x)}{[2,2,3]\left(x^{2}+1\right)\left(x^{2}-x+1\right)},
\end{aligned}
$$

which is related inversely to the growth function $f_{(3,3,6)}(x)$ according to (see (4-11))

$$
\frac{1}{f_{(3,3,6)(x)}}=\frac{1-x}{1+x}\left\{1-\frac{x}{2} H_{(3,3,6)}(x)\right\} \text {. }
$$

Lemma 9 The polynomial $p(x)$ in (5-1) given by

$$
p(x)=x^{6}+2 x^{5}+2 x^{4}+3 x^{3}+2 x^{2}+2 x+1
$$

is a Salem polynomial.

Proof Obviously, $p( \pm 1) \neq 0$. Furthermore, $p(x)$ is a palindromic monic polynomial of (even) degree six over the integers. By an adaption of a result of Kempner (see Zehrt and Zehrt-Liebendörfer [23, Proposition 1]), we conclude that $p(x)$ is a Salem polynomial in the following way. Consider the polynomial

$$
q(x)=(x-i)^{6} p\left(\frac{x+i}{x-i}\right)=13 x^{6}-37 x^{4}+15 x^{2}+1 \in \mathbb{Z}[x],
$$


which is of degree six and even. There is the following equivalence between the roots of $p$ and $q$.

(a) $\quad p$ has $2 k$ roots on the unit circle if and only if $q$ has $k$ positive real roots.

(b) $p$ has $2 l$ real roots if and only if $q$ has $l$ positive imaginary roots.

Let us show that $q$ has two positive real roots and one positive imaginary root as follows (see Rotman [17, Classical Formulas], for example). By substituting $y=x^{2}$ into (5-3), we see that the cubic equation $13 y^{3}-37 y^{2}+15 y+1=0$ has positive discriminant and therefore three distinct real roots. These roots are given by explicit formulas, and their inspection shows that exactly one root is negative. Since $y=x^{2}$, the equation $13 x^{6}-37 x^{4}+15 x^{2}+1=0$ has two positive real roots and one positive imaginary root. Therefore, $p(x)$ is a Salem polynomial.

\subsection{The two-dimensional case}

The method which we developed in order to prove that the Coxeter group $(3,3,6)$ has minimal growth rate among all non-cocompact cofinite Coxeter groups in $\mathbb{H}^{3}$ can be applied to the two-dimensional case as well. This approach gives an alternative proof of the following result of Floyd [4, page 482], which is more elementary, without reference to Pisot polynomials and the respective minimality result of Smyth (see Section 3.2).

Proposition Among all hyperbolic Coxeter groups with non-compact fundamental polygon of finite volume in $\mathbb{H}^{2}$, the triangle group $(3, \infty)$ has minimal growth rate.

Proof Denote by $P \subset \mathbb{H}^{2}$ a fundamental polygon of a hyperbolic Coxeter group $(G, S)$ such that $P$ is non-compact but of finite volume. Let $\Omega_{0}=\Omega_{f} \cup \Omega_{\infty}$ be the set of vertices partitioned into the set $\Omega_{f}$ of finite vertices and the set $\Omega_{\infty}$ of ideal vertices of $P$. Let $\mathfrak{f}_{0}=\left|\Omega_{0}\right|$ and $\mathfrak{f}_{0}^{\infty}=\left|\Omega_{\infty}\right|$. It is a particular feature in two dimensions that the number of vertices $\mathfrak{f}_{0}$ equals the number of edges $\mathfrak{f}_{1}=|S|$ and that $\mathfrak{f}_{0}-\mathfrak{f}_{0}^{\infty}$ equals the number of (positive) angles of $P$ which are of the form $\frac{\pi}{k}$ for integers $k \geq 2$. Denote by $2 k_{v}$ the order of the stabiliser $D_{2}^{k}$ of the vertex $v \in \Omega_{f}$. By the formula (3-3) and (3-4) of Steinberg and Solomon, and by Table 3, we can derive the following expression for the growth function $f:=f_{S}$ of $G=(G, S)$.

$$
\begin{aligned}
\frac{1}{f\left(x^{-1}\right)} & =1-\frac{\mathfrak{f}_{0}}{[2]}+\sum_{v \in \Omega_{f}} \frac{1}{\left[2, k_{v}\right]} \\
& =1-\frac{1}{[2]}\left\{\mathfrak{f}_{0}^{\infty}+\sum_{v \in \Omega_{f}}\left(1-\frac{1}{\left[k_{v}\right]}\right)\right\} \\
& =1-\frac{1}{1+x}\left\{\mathfrak{f}_{0}^{\infty}+x \sum_{v \in \Omega_{f}} \frac{\left[k_{v}-1\right]}{\left[k_{v}\right]}\right\} .
\end{aligned}
$$


The passage $x \mapsto x^{-1}$ and the property (3-5) yield

$$
\frac{1}{f(x)}=1-\frac{x}{[2]}\left\{\mathfrak{f}_{0}^{\infty}+\sum_{v \in \Omega_{f}} \frac{\left[k_{v}-1\right]}{\left[k_{v}\right]}\right\}=: 1-\frac{x}{[2]} H(x),
$$

where we put

$$
H(x):=\mathfrak{f}_{0}^{\infty}+\sum_{v \in \Omega_{f}} \frac{\left[k_{v}-1\right]}{\left[k_{v}\right]}
$$

so that

$$
H_{(3, \infty)}(x)=1+\frac{1}{[2]}+\frac{[2]}{[3]} .
$$

As in the three-dimensional case, we shall prove the Proposition by showing that each Coxeter group $G$ different from the triangle group $(3, \infty)$ satisfies (see $(5-5)$ )

$$
\frac{1}{f(x)}<\frac{1}{f_{(3, \infty)}(x)}=\frac{1-x^{2}-x^{3}}{[2,2,3]} \text { for } x \in\left(0,1 / \tau_{(0, \infty)}\right],
$$

which, by (4-16), (5-6) and (5-7), is equivalent to the verification of

$$
H(x)=\mathfrak{f}_{0}^{\infty}+\sum_{v \in \Omega_{f}} h_{k_{v}}(x)>1+\frac{1}{[2]}+\frac{[2]}{[3]}=H_{(3, \infty)}(x)
$$

on the interval $\left(0,1 / \tau_{(0, \infty)}\right]$. We have $f_{0}^{\infty} \geq 1$, and by Lemma 8 , each term $h_{k}(x):=$ $h_{k_{v}}(x)$ satisfies $0<h_{k}(x)<h_{k+1}<1$ on $(0,1)$. These properties and the realisation condition for hyperbolic Coxeter polygons with $\mathfrak{f}_{0}$ vertices and $\mathfrak{f}_{0}-\mathfrak{f}_{0}^{\infty}$ positive angles $\pi / k_{v}, v \in \Omega_{f}$, that is,

$$
\sum_{v \in \Omega_{f}} \frac{1}{k_{v}}<\mathfrak{f}_{0}-2
$$

shows that a minimiser of the functions $H(x)$ on $(0,1)$ must have $f_{0}^{\infty}=1$ as well as $\mathfrak{f}_{0}=3$ with $k_{v_{1}}=2$ and $k_{v_{2}}=3$. These conditions are fulfilled only by the triangle group $(3, \infty)$.

Remark By the above Proposition, and Sections 3.2 and 5.1, the growth rate $\tau$ of any hyperbolic Coxeter group $G$ with non-compact fundamental polygon of finite volume in $\mathbb{H}^{2}$ satisfies $\tau \geq \tau_{(3, \infty)}=\alpha_{S} \simeq 1.324718>1.296466 \simeq \tau_{*}=\tau_{(3,3,6)}$, with equality $\tau=\tau_{(3, \infty)}$ if and only if $G=(3, \infty)$. 


\section{References}

[1] R Charney, M Davis, Reciprocity of growth functions of Coxeter groups, Geom. Dedicata 39 (1991) 373-378 MR1123152

[2] HS M Coxeter, Discrete groups generated by reflections, Ann. of Math. 35 (1934) 588-621 MR1503182

[3] H S M Coxeter, W O J Moser, Generators and relations for discrete groups, fourth edition, Ergeb. Math. Grenzgeb. 14, Springer, Berlin (1980) MR562913

[4] W J Floyd, Growth of planar Coxeter groups, $P$ V numbers, and Salem numbers, Math. Ann. 293 (1992) 475-483 MR1170521

[5] P de la Harpe, Groupes de Coxeter infinis non affines, Exposition. Math. 5 (1987) 91-96 MR880259

[6] E Hironaka, The Lehmer polynomial and pretzel links, Canad. Math. Bull. 44 (2001) 440-451 MR1863636

[7] N W Johnson, R Kellerhals, J G Ratcliffe, S T Tschantz, The size of a hyperbolic Coxeter simplex, Transform. Groups 4 (1999) 329-353 MR1726696

[8] I M Kaplinskaja, The discrete groups that are generated by reflections in the faces of simplicial prisms in Lobačevskĭ spaces, Mat. Zametki 15 (1974) 159-164 MR0360858

[9] R Kellerhals, A Kolpakov, The minimal growth rate of cocompact Coxeter groups in hyperbolic 3-space, to appear in Canadian J. Math.

[10] R Kellerhals, G Perren, On the growth of cocompact hyperbolic Coxeter groups, European J. Combin. 32 (2011) 1299-1316 MR2838016

[11] A Kolpakov, Deformation of finite-volume hyperbolic Coxeter polyhedra, limiting growth rates and Pisot numbers, European J. Combin. 33 (2012) 1709-1724 MR2950475

[12] Y Komori, Y Umemoto, The growth functions of noncompact 3-dimensional hyperbolic Coxeter groups with 4 and 5 generators, preprint (2012)

[13] Y Komori, Y Umemoto, On the growth of hyperbolic 3-dimensional generalized simplex reflection groups, Proc. Japan Acad. Ser. A Math. Sci. 88 (2012) 62-65 MR2912844

[14] J-L Koszul, Lectures on hyperbolic Coxeter groups, lecture notes, University of Notre Dame (1967)

[15] R Meyerhoff, The cusped hyperbolic 3-orbifold of minimum volume, Bull. Amer. Math. Soc. 13 (1985) 154-156 MR799800

[16] W Parry, Growth series of Coxeter groups and Salem numbers, J. Algebra 154 (1993) 406-415 MR1206129

[17] J Rotman, Galois theory, second edition, Universitext, Springer, New York (1998) MR1645586 
[18] C J Smyth, On the product of the conjugates outside the unit circle of an algebraic integer, Bull. London Math. Soc. 3 (1971) 169-175 MR0289451

[19] L Solomon, The orders of the finite Chevalley groups, J. Algebra 3 (1966) 376-393 MR0199275

[20] R Steinberg, Endomorphisms of linear algebraic groups, Memoirs of the American Mathematical Society 80, American Mathematical Society (1968) MR0230728

[21] ̀ B Vinberg, Hyperbolic groups of reflections, Uspekhi Mat. Nauk 40 (1985) 29-66, 255 MR783604

[22] ̇̀ B Vinberg, O V Shvartsman, Discrete groups of motions of spaces of constant curvature, from: “Geometry, II”, Encyclopaedia Math. Sci. 29, Springer, Berlin (1993) 139-248 MR1254933

[23] T Zehrt, C Zehrt-Liebendörfer, The growth function of Coxeter garlands in $\mathbb{H}^{4}$, Beitr. Algebra Geom. 53 (2012) 451-460 MR2971753

Department of Mathematics, University of Fribourg

CH-1700 Fribourg, Switzerland

ruth.kellerhals@unifr.ch

Received: 12 July 2012 Revised: 30 November 2012 